\title{
Adverse Events by Sedation Type in Gastrointestinal Endoscopy
}

\author{
Joon Sung Kim and Byung-Wook Kim \\ Division of Gastroenterology, Department of Internal Medicine, Incheon St. Mary's Hospital, The Catholic University of Korea College of \\ Medicine, Incheon, Korea
}

See "Association between Type of Sedation and the Adverse Events Associated with Gastrointestinal Endoscopy: An Analysis of 5 Years' Data from a Tertiary Center in the USA" by Basavana Goudra, Ahmad Nuzat, Preet Mohinder Singh, et al., on page 161-169.

Due to its early onset of action, short half-life, and short recovery period, in recent years, propofol has been increasingly used for sedation during endoscopic procedures. ${ }^{1}$ Although it is generally associated with good hemodynamic stability, dose-dependent respiratory depression and a decrease in the blood pressure and heart rates can occur. ${ }^{2}$ An impaired cardiac function has been reported to potentiate the drug's effect, and no pharmacological antagonist is currently available for it. This has suggested that the use of propofol was associated with a higher rate of cardiopulmonary adverse events.

While in the United States propofol is generally administered by anesthesia specialists, it is administered by endoscopists in Korea. ${ }^{3}$ Concerns about the safety of endoscopist-directed propofol (EDP) have been voiced. However, studies have reported it to be quite safe. In a study involving 82,620 endoscopic procedures, no severe adverse effects associated with EDP were found. ${ }^{4}$ In another study, 638 colonoscopies and 181 upper endoscopies were performed under EDP. ${ }^{5}$ Hypotension was found in 218 patients (27\%), while hypoxemia occurred in 75 patients (9\%). However, all episodes of hypotension and hypoxemia were transient, with no need for assisted ventilation. Most recently, a meta-analysis of 2,518

Received: February 10, 2017 Accepted: February 24, 2017

Correspondence: Byung-Wook Kim

Division of Gastroenterology, Department of Internal Medicine, Incheon St. Mary's Hospital, College of Medicine, The Catholic University of Korea, 56 Dongsu-ro, Bupyeong-gu, Incheon 21431, Korea

Tel: +82-32-280-5052, Fax: +82-32-280-5987, E-mail: gastro@catholic.ac.kr

(c) This is an Open Access article distributed under the terms of the Creative Commons Attribution Non-Commercial License (http://creativecommons.org/ licenses/by-nc/3.0) which permits unrestricted non-commercial use, distribution, and reproduction in any medium, provided the original work is properly cited. patients from 27 studies reported that propofol sedation presented a similar risk of cardiopulmonary adverse events to that from traditional agents. ${ }^{6}$

The article in this issue of Clinical Endoscopy by Goudra et al. ${ }^{7}$ is based on 73,029 endoscopic procedures. Sedation was provided by either propofol or intravenous conscious sedation (IVCS). Propofol was administered by a certified anesthesiologist or a certified nurse under the supervision of an anesthesiologist. IVCS was administered by a nurse under supervision from the endoscopist. Bleeding was found to be the most common adverse event, followed by cardiorespiratory arrest and post-procedural pain. Unlike in previous studies, the frequency of most adverse events was significantly higher in patients receiving propofol than in those receiving IVCS. Automatic regression modeling showed that the type of sedation, the American Society of Anesthesiologist (ASA) physical status classification, and the procedure type were predictors of immediate life-threatening complications. Interestingly, the rate of adverse events was higher in the propofol group even though the administration was performed by the anesthesiologist or under the supervision of the anesthesiologist. Although the anesthesiologist directed administration of sedatives would seem likely to decrease the development of complications, recent studies have suggested an increase in the number of complications when sedatives are administered by anethesiologists. ${ }^{8,9}$ In addition, the administration of propofol by anesthesia specialists would inevitably increase the cost of endoscopic procedures. Currently, no studies have compared propofol administration by an endoscopist to that by an anesthesiologist. ${ }^{6}$ Several cases of propofol-related deaths during endoscopy are reported every year in Korea. As Voltaire said, 
"It is the danger that is the least expected that will come to us the soonest". Endoscopists using propofol for sedation during endoscopy should be familiar with the management of adverse events. In addition, propofol sedation should be used with caution in high-risk patients or in complex procedures with long durations.

Conflicts of Interest

The authors have no financial conflicts of interest.

\section{REFERENCES}

1. Cohen LB, Wecsler JS, Gaetano JN, et al. Endoscopic sedation in the United States: results from a nationwide survey. Am J Gastroenterol 2006;101:967-974.

2. Trapani G, Altomare C, Liso G, Sanna E, Biggio G. Propofol in anesthesia. Mechanism of action, structure-activity relationships, and drug delivery. Curr Med Chem 2000;7:249-271.
3. Kim EH, Lee SK. Endoscopist-directed propofol: pros and cons. Clin Endosc 2014;47:129-134.

4. Heuss LT, Froehlich F, Beglinger C. Changing patterns of sedation and monitoring practice during endoscopy: results of a nationwide survey in Switzerland. Endoscopy 2005;37:161-166.

5. Cohen LB, Dubovsky AN, Aisenberg J, Miller KM. Propofol for endoscopic sedation: a protocol for safe and effective administration by the gastroenterologist. Gastrointest Endosc 2003;58:725-732.

6. Wadhwa V, Issa D, Garg S, Lopez R, Sanaka MR, Vargo JJ. Similar risk of cardiopulmonary adverse events between propofol and traditional anesthesia for gastrointestinal endoscopy: a systematic review and meta-analysis. Clin Gastroenterol Hepatol 2017;15:194-206.

7. Goudra B, Nuzat A, Singh PM, Borle A, Carlin A, Gouda G. Association between type of sedation and the adverse events associated with gastrointestinal endoscopy: an analysis of 5 years' data from a tertiary center in the USA. Clin Endosc 2017;50:161-169.

8. Cooper GS, Kou TD, Rex DK. Complications following colonoscopy with anesthesia assistance: a population-based analysis. JAMA Intern Med 2013;173:551-556.

9. Wernli KJ, Brenner AT, Rutter CM, Inadomi JM. Risks associated with anesthesia services during colonoscopy. Gastroenterology 2016;150:888894. 